\title{
Changes in amplitude of somatosensory evoked potentials of elderly by mechanical vibratory stimulation applied to tibialis anterior tendon
}

\author{
Huigyun Kim ${ }^{1}$, Kiyoung Kwak ${ }^{2}$, Dongwook Kim ${ }^{3}$ \\ ${ }^{1,2}$ Department of Healthcare Engineering, Graduate School, Chonbuk National University, \\ Jeonju, Republic of Korea \\ ${ }^{3}$ Department of Biomedical Engineering, College of Engineering, Chonbuk National University, \\ Jeonju, Republic of Korea \\ ${ }^{3}$ Research Center of Healthcare and Welfare Instrument for Aged, Chonbuk National University, \\ Jeonju, Republic of Korea \\ ${ }^{3}$ Corresponding author \\ E-mail: ${ }^{1}$ hekun1249@naver.com, ${ }^{2} k y k w a k 86 @ n a t e . c o m,{ }^{3}$ biomed@jbnu.ac.kr
}

Received 28 May 2017; accepted 29 May 2017

DOI https://doi.org/10.21595/vp.2017.18687

Check for updates

\begin{abstract}
In this study, the researchers examined the changes that took place in the somatosensory cortex area in the brain when subthreshold mechanical vibratory stimulation was applied to the lower limb of the elderly. For this, a subthreshold vibratory stimulation of $190 \mathrm{~Hz}$ as applied to the tibialis anterior tendon, and the Electroencephalography was measured. In order to examine the changes in the somatosensory cortex, the amplitude of the Somatosensory Evoked Potentials (SEPs) was analyzed. When the subthreshold vibratory stimulation was applied to the tibialis anterior tendon, the SEPs amplitude was increased more than the amplitude under none stimulation setting. This result means that the subthreshold mechanical vibratory stimulation may affect the nervous system of the elderly.
\end{abstract}

Keywords: elderly, subthreshold, mechanical vibratory stimulation, SEPs, somatosensory evoked potentials.

\section{Introduction}

The aging process weakens the performance of the nervous and musculoskeletal system. And, this affect the degradation of the quality of life [1]. Frolkis, et al. reported that, as the aging process continues, the transmission speed in the nerves in the central nervous system is decreased [2]. MacLennan, et al., reported that aging influences the number, size, and tensile strength of the muscular tissues in the musculoskeletal system, affecting the coordination between the muscles and the nerves [3]. The changes in the nervous and musculoskeletal system significantly affect the motions performed in the daily life of the elderly, such as walking, sitting and standing, and climbing stairs $[4,5]$.

There have been a number of studies to improve the quality of life of the elderly using vibratory stimuli. Bosco, et al. reported that mechanical vibratory stimuli improved the muscle strength and balance, positively influencing the elderly in the face of the factors such as fall injuries or fractures [6]. Runge, et al. reported that the vibratory stimuli may enhance the muscle strength of the elderly [7]. In most studies, the researchers examined the influence of the vibratory stimuli on the musculoskeletal system of the elderly. And, further studies on the influence of the vibratory stimuli on the nervous systems of the elderly are needed. Therefore, the purpose of this study is to explore the changes in the somatosensory cortex area of the brain when subthreshold strength vibratory stimuli is applied on the lower limbs of the elderly through SEPs, based on the precedent studies where the perception threshold intensity based on the frequency of the vibration [8].

\section{Methods}

For this study, 10 male subjects who were 65 or up and without any experience with 
experiments or problems with physical activities were selected and included. The research was approved by the IRB of Chonbuk National University (IRB File No. JBNU 2015-06-012). The devices used to apply the vibratory stimuli were a function generator (AFG-2125, GOOD WILL INSTRUMENT Co., Ltd) and a linear actuator (DMJBRN0934AA, SAMSUNG ELECTRO-MECHANICS Co., Ltd). Using a rubber band, the vibrator was attached to the tibialis anterior tendon.

Based on the preceding studies on the perception threshold intensity of vibration frequency [8], a frequency of $190 \mathrm{~Hz}$ was selected and applied to the tibialis anterior tendon. Before the SEPs test, the vibration perception threshold was measured for each participant. The vibratory stimulation intensity was divided into none stimulation, threshold, and sub threshold intensity. At each intensity, 100 cycles of vibration were applied at $500 \mathrm{~ms}$ intervals. The sequence of applying the vibration was randomly selected.

Based on the internationally certified 10-20 system for measuring the brain signals, EEG electrode were attached to the $\mathrm{C} 3$ and $\mathrm{C} 4$ area, which were related to the somatosensory cortex. Also, the researchers used EEG collection system called BrainAmp (Brain Products, Brain Products GmbH, Germany), and EEG analysis system called BESA (BESA GmbH, Germany). The sampling rate of the measurement was $1000 \mathrm{~Hz}$. The bandwidth filter used was $0.1-70 \mathrm{~Hz}$. A notch filter was used in order to remove noise. The brainwave and the trigger signal for vibration were synchronized.

From the EEG data gathered, SEPs were extracted. With regard to the SEPs, based on the trigger signal for vibration, the negative peak at $100-150 \mathrm{~ms}$ and the positive peak at 220-350 ms were extracted [9]. And, the change in the amplitude of the SEPs under different conditions were compared.

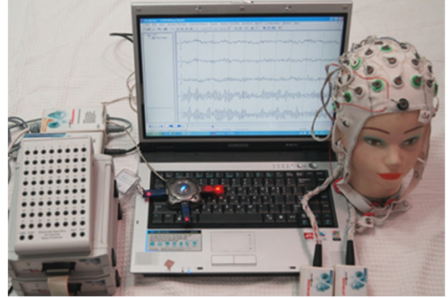

a)

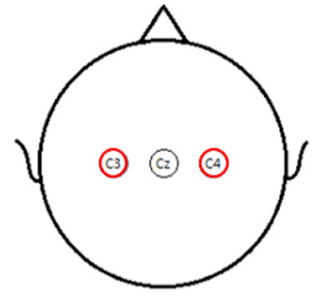

b)

Fig. 1. a) EEG collection and analysis system, b) EEG electrode attaching position

\section{Results}

The changes in the amplitude of the SEPs due to a mechanical vibratory stimulation of $190 \mathrm{~Hz}$ applied to the tibialis anterior tendon of the elderly subject were as follows; Fig. 2 shows that the amplitude increased more when the threshold intensity stimulation was applied than when the vibratory stimulation was not applied. The amplitude of the negative and positive peaks in $\mathrm{C} 3$ and the negative peak in $\mathrm{C} 4$ increased significantly more compared to the none-stimulation condition.

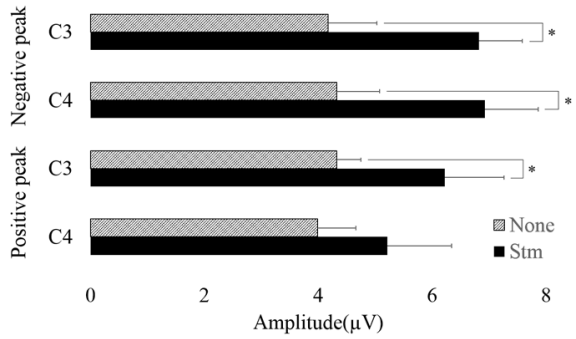

Fig. 2. Amplitude $(\mu \mathrm{V})$ changes of SEPs stimulation and none stimulation.

None: None stimulation, Stm: stimulation, ${ }^{*}: P<0.05$. None stimulation versus stimulation 
Fig. 3 shows the changes in the amplitude of the Negative peak of SEPs based on the intensity of the vibratory stimulation applied to the tibialis anterior tendon of the elderly subjects. The amplitude of the SEPs showed a tendency to increase with vibration of subthreshold intensity compared to none stimulation condition. Especially, in both $\mathrm{C} 3$ and $\mathrm{C} 4$ area, the amplitude at an intensity that was $80 \%$ or more of the threshold intensity increased more compared to the none stimulation condition, and the difference was statistically significant.

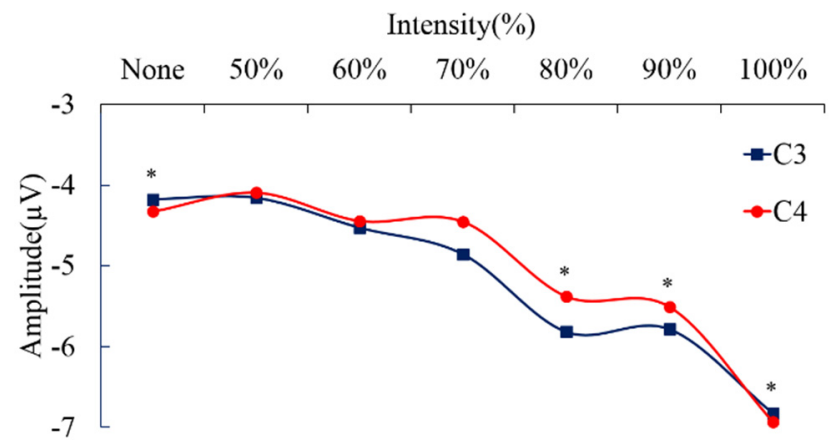

Fig. 3. Amplitude $(\mu \mathrm{V})$ changes of negative peak of SEPs according to vibration intensity (\%);

${ }^{*}: P<0.05$. None stimulation versus stimulation intensity

Fig. 4 shows the changes in the amplitude of the Positive peak of SEPs based on the intensity of the vibratory stimulation applied to the tibialis anterior tendon of the elderly subjects. The amplitude of the SEPs showed a tendency to increase with vibration of subthreshold intensity compared to none stimulation condition. Especially, in both $\mathrm{C} 3$ and $\mathrm{C} 4$ area, the amplitude at an intensity that was $80 \%$ or more of the threshold intensity increased more compared to the none stimulation condition, and the difference was statistically significant.

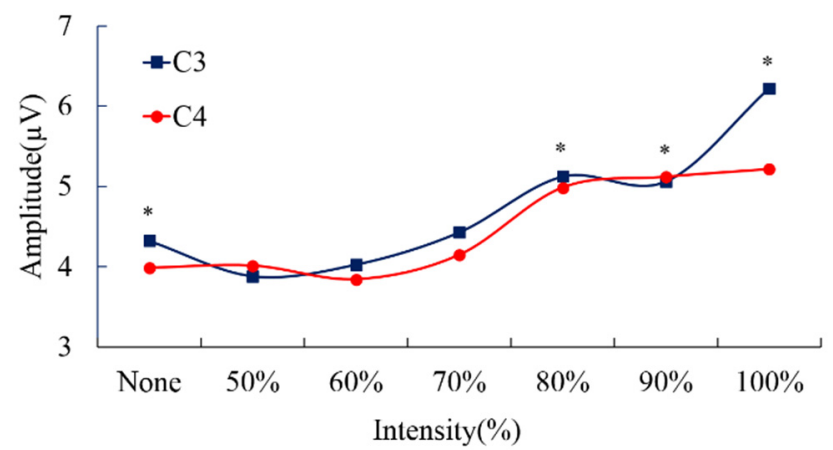

Fig. 4. Amplitude $(\mu \mathrm{V})$ changes of positive peak of SEPs according to vibration intensity (\%);

${ }^{*}: P<0.05$. None stimulation versus stimulation intensity $(\%)$

In the result mentioned above, the amplitude of the SEPs of the mechanical vibratory stimulation of threshold intensity applied to the tibialis anterior tendon increased more than that under the none-stimulation condition. Such a result indicates that the vibratory stimulation may affect the nervous system of the elderly. Also, with the vibratory stimulation of subthreshold intensity, both of $\mathrm{C} 3$ and $\mathrm{C} 4$ showed that the SEPs amplitude increased more than that under the nine stimulation condition, while significance was observed with the amplitude of the $80 \%$ or higher intensity. Such a result shows that the vibratory stimulation of subthreshold intensity, which are free of the discomfort and interference with the sensory system $[10,11]$ experienced with the vibration of the individual perception threshold intensity or higher intensity, may still affect the nervous system of the elderly. 


\section{Conclusions}

In this study, the researchers examined the changes that took place in the somatosensory cortex area in the brain when mechanical vibratory stimulation of perception subthreshold intensity was applied to the lower limb of the elderly through SEPs. When the vibratory stimulation of subthreshold intensity was applied to the tibialis anterior tendon, the SEPs amplitude was increased more than the amplitude under none simulation setting. The result showed that the vibratory stimulation of subthreshold intensity may affect the nervous system of the elderly. If the findings in this study are applied to the elderly whose balancing ability is weakened, it would be possible to contribute to the enhancement of their quality of life by improving the balancing capability of them.

\section{Acknowledgements}

This work was supported by the National Research Foundation of Korea (NRF) Grant funded by the Korea Government (MSIP) (NRF-2014R1A2A1A11053073).

\section{References}

[1] Degens H. Age-related skeletal muscle dysfunction: causes and mechanisms. Journal of Musculoskeletal and Neuronal Interaction, Vol. 7, Issue 3, 2007, p. 246-252.

[2] Frolkis V. V., Martynenko O. A., Zamostyan V. P. Aging of the neuromuscular apparatus. Gerontology, Vol. 22, Issue 4, 1976, p. 244-279.

[3] MacLennan W. J., Hall M. R., Timothy J. I., Robinson M. Is weakness in old age due to muscle wasting? Age Ageing, Vol. 9, Issue 3, 1980, p. 188-192.

[4] Janssen I., Heymsfield S. B., Ross R. Low relative skeletal muscle mass (sarcopenia) in older persons is associated with functional impairment and physical disability. Journal of the American Geriatrics Society, Vol. 50, Issue 5, 2002, p. 889-896.

[5] Vincent K. R., Braith R. W., Feldman R. A., et al. Resistance exercise and physical performance in adults aged 60 to 83. Journal of the American Geriatrics Society, Vol. 50, Issue 6, 2002, p. 664-672.

[6] Bosco C., Cardinale M., Tsarpela O., et al. The influence on whole body vibration on jumping performance. Biology of Sport, Vol. 15, Issue 3, 1998, p. 157-164.

[7] Runge M., Rehfeld G., Resnicek E. Balance training and exercise in geriatric patients. Journal of Musculoskeletal and Neuronal Interaction, Vol. 1, Issue 1, 2000, p. 54-58.

[8] Kwak K. Y., Kim H. G., Kim D. W. Variation of ankle biomechanical property according to vibro-perception threshold and vibration frequency. Biomedical Engineering Letters, Vol. 6, Issue 1, 2016, p. 16-25.

[9] Reinacher M., Becker R., Villringer A., Ritter P. Oscillatory brain states interact with latecognitive components of the somatosensory evoked potential. Journal of Neuroscience Methods, Vol. 183, Issue 1, 2008, p. 49-56.

[10] Pope M. H., Pope Magn M. H., Magnusson M., Wilder D. G. Low back pain and whole body vibration. Clinical Orthopaedics and Related Research, Vol. 354, Issue 1, 1998, p. 241-248.

[11] Simeonov P., Hsiao H., Powers J., et al. Postural stability effects of random vibration at the feet of construction workers in simulated elevation. Applied Ergonomics, Vol. 42, Issue 5, 2011, p. 672-681. 